\title{
Strong convergence by the shrinking effect of two half-spaces and applications
}

\author{
Muhammad Aqeel Ahmad Khan ${ }^{1,2^{*}}$ and Hafiz Fukhar-ud-din ${ }^{2,3}$
}

\author{
"Correspondence: \\ itsakb@hotmail.com \\ 'Department of Mathematics, \\ Technische Universitat Darmstadt, \\ Schlossgartenstrasse 7, Darmstadt, \\ 64289, Germany \\ ${ }^{2}$ Department of Mathematics, The \\ Islamia University of Bahawalpur, \\ Bahawalpur, 63100, Pakistan \\ Full list of author information is \\ available at the end of the article
}

\begin{abstract}
This paper provides a new hybrid-type shrinking projection method for strong convergence results in a Hilbert space. The paper continues - by utilizing the proposed hybrid algorithm - with a strong convergence towards an approximate common element of the set of solutions of a finite family of generalized equilibrium problems and the set of common fixed points of two finite families of $k$-strict pseudo-contractions in a Hilbert space. Comparatively, our results improve and extend various results announced in the current literature.
\end{abstract}

MSC: Primary 47H05; 47H09; secondary 49H05

Keywords: nonexpansive map; strict pseudo-contraction; shrinking projection method; CQ-method; equilibrium problem; $\delta$-inverse strongly monotone map

\section{Introduction}

Let $H$ be a real Hilbert space equipped with the inner product $\langle\cdot, \cdot\rangle$ and the induced norm $\|\cdot\|$ and let $C$ be a nonempty closed convex subset of $H$. A map $T: C \rightarrow C$ is said to be (i) Lipschitzian if $\|T x-T y\| \leq L\|x-y\|$ for some $L>0$ and for all $x, y \in C$; (ii) nonexpansive if $\|T x-T y\| \leq\|x-y\|$ for all $x, y \in C$; (iii) $k$-strict pseudo-contraction if there exists a constant $k \in[0,1)$ such that

$$
\|T x-T y\|^{2} \leq\|x-y\|^{2}+k\|(I-T) x-(I-T) y\|^{2} \quad \text { for all } x, y \in C
$$

Denote by $F(T)$ the set of all fixed points of $T$.

In 1967, Browder and Petryshyn [1] introduced the class of strict pseudo-contractions as an important generalization of the class of nonexpansive maps. Clearly, $T$ is nonexpansive if and only if $T$ is a 0 -strict pseudo-contraction. Moreover, $T$ is said to be pseudocontraction if $k=1$ and a strong pseudo-contraction if there exists a positive constant $\lambda \in(0,1)$ such that $T-\lambda I$ is a pseudo-contraction. Therefore, the class of $k$-strict pseudocontractions falls into the one between the classes of nonexpansive maps and pseudocontractions. We further remark that the class of strong pseudo-contractions is independent of the class of $k$-strict pseudo-contractions. If $T$ is a $k$-strict pseudo-contraction, then $T$ satisfies the Lipschitz condition

$$
\|T x-T y\| \leq \frac{1+k}{1-k}\|x-y\| \quad \text { for all } x, y \in C .
$$

C) 2013 Khan and Fukhar-ud-din; licensee Springer. This is an Open Access article distributed under the terms of the Creative Commons Attribution License (http://creativecommons.org/licenses/by/2.0), which permits unrestricted use, distribution, and reproduction in any medium, provided the original work is properly cited. 
Recent developments in fixed point theory reflect that an iterative construction of fixed points of nonlinear maps is an active area of research. Note that the class of $k$-strict pseudo-contractions is prominent among the classes of nonlinear maps in the current literature. Although strict pseudo-contractions have more powerful applications than nonexpansive maps for solving inverse problems, iterative algorithms for strict pseudocontractions are far less developed than those for nonexpansive maps.

In fixed point theory, various iterative schemas for computing approximate fixed points of nonlinear maps have been proposed and analyzed. Such iterative schemas can be compared w.r.t. their efficiency and convergence properties. In many situations, strong convergence of an iterative algorithm of a nonlinear map is much more desirable than weak convergence. Obviously, a trivial choice for approximation is the classical Picard algorithm (i.e., $x_{n+1}=T^{n}\left(x_{0}\right)$ ). On the other hand, if we take $X=\mathbb{R}, C:=[0,1], T(x):=1-x$ and $x_{0}:=0$, then the Picard algorithm alternates between 0 and 1 and does not converge to the fixed point $\frac{1}{2}$. The classical Mann algorithm, which prevails the Picard algorithm, exhibits weak convergence even in the setting of a Hilbert space. Moreover, Chidume and Mutangadura [2] constructed an example for a Lipschitz pseudo-contraction with a unique fixed point for which the Mann algorithm fails to converge. These facts indicate that the iterative schemas should be modified for the desirable strong convergence properties.

The hybrid projection algorithm in mathematical programming was introduced by Haugazeau [3] in 1968. Later, many researchers studied the hybrid projection method and its various modifications for strong convergence results. In 2000, Solodov and Svaiter [4] established a strong convergence result for finding zeros of maximal monotone operators. They proposed and analyzed the following algorithm:

$$
\begin{aligned}
& v_{k} \in A y_{k}, \quad v_{k}+\mu_{k}\left(y_{k}-x_{k}\right)=\epsilon_{k} ; \\
& \left\|\epsilon_{k}\right\| \leq \sigma \max \left\{\left\|v_{k}\right\|, \mu_{k}\left\|y_{k}-x_{k}\right\|\right\} ; \\
& H_{k}=\left\{z \in H:\left\langle z-y_{k}, v_{k}\right\rangle \leq 0\right\} ; \\
& W_{k}=\left\{z \in H:\left\langle x_{k}-z, x_{0}-x_{k}\right\rangle \geq 0\right\} ; \\
& x_{k+1}=P_{H_{k} \cap W_{k}} x_{0} .
\end{aligned}
$$

In fact, algorithm (1.1) enforces strong convergence by combining the classical proximal point algorithm with simple projection steps onto intersection of two half-spaces $H_{k}$ and $W_{k}$ containing the solution set. Inspired by the seminal work of Solodov and Svaiter [4], Nakajo and Takahashi [5] proposed the following hybrid method for nonexpansive maps in Hilbert spaces:

$$
\begin{aligned}
& x_{0} \in C \quad \text { chosen arbitrarily, } \\
& y_{k}=\alpha_{k} x_{k}+\left(1-\alpha_{k}\right) T x_{k}, \\
& C_{k}=\left\{z \in C:\left\|y_{k}-z\right\| \leq\left\|x_{k}-z\right\|\right\}, \\
& Q_{k}=\left\{z \in C:\left\langle x_{n}-z, x-x_{n}\right\rangle \geq 0\right\}, \\
& x_{k+1}=P_{C_{k} \cap Q_{k}} x_{0} .
\end{aligned}
$$

They showed that algorithm (1.2) converges strongly to $P_{F(T)} x$ under some appropriate conditions. Moreover, algorithm (1.2) is also known as the CQ-method for the Mann al- 
gorithm because, at each step, the Mann algorithm is used to construct the sets $C_{k}$ and $Q_{k}$ which are in turn used to construct the next iterate of $x_{k+1}$ and hence the name.

In 2008, Takahashi et al. [6] introduced another type of the hybrid method which guarantees strong convergence by the shrinking effect of a sequence of closed convex sets $\left\{C_{k+1}\right\}$. More precisely, their algorithm reads as follows:

$$
\begin{aligned}
& x_{0} \in C \quad \text { chosen arbitrarily where } C_{0}=C, \\
& y_{k}=\alpha_{k} x_{k}+\left(1-\alpha_{k}\right) T x_{k}, \\
& C_{k+1}=\left\{z \in C_{k}:\left\|z-y_{k}\right\| \leq\left\|z-x_{k}\right\|\right\}, \\
& x_{k+1}=P_{C_{k+1}} x_{0}, \quad k \geq 0 .
\end{aligned}
$$

Very recently, Dong et al. [7] proposed a shrinking projection method similar to (1.3) for nonexpansive maps in a Hilbert space setting. They, in fact, established a strong convergence result by the shrinking effect of one of the half-spaces as defined in (1.2), namely $Q_{k}$.

For $i=1,2,3, \ldots, N$, let $A_{i}: C \rightarrow H$ be a finite family of nonlinear maps and $f_{i}: C \times C \rightarrow$ $\mathbb{R}$ (the set of reals) be a finite family of bifunctions. A generalized equilibrium problem is to find the set

$$
\operatorname{GEP}\left(f_{i}\right)=\left\{x \in C: f_{i}(x, y)+\left\langle A_{i} x, y-x\right\rangle \geq 0\right\} \quad \text { for all } y \in C \text {. }
$$

Note that if $A_{i} \equiv 0$ and $f_{i}(x, y)=f(x, y)$ for all $i \geq 1$, then the problem (1.4) reduces to the classical equilibrium problem $\operatorname{EP}(f)$. That is, to find $x \in C$ such that $f(x, y) \geq 0$. Moreover, if $f_{i}(x, y) \equiv 0$ and $A_{i}=A$ for all $i \geq 1$, then the problem (1.4) reduces to the classical variational inequality problem $\operatorname{VI}(C, A)$. That is, to find $x \in C$ such that $\langle A x, y-x\rangle \geq 0$.

Equilibrium problems provide a unified approach to address a variety of problems arising in various disciplines of science. The problem (1.4) is very general in the sense that it includes - as special cases - optimization problem, minimax problem, variational inequality problem, Nash equilibrium problem in noncooperative games and others; see, for instance, [8-10]. Combettes and Hirstoaga [9] introduced an iterative method to find an approximate solution of $\operatorname{EP}(f)$. Since then, numerous algorithms have been analyzed to find a common element of the set of solutions of $\operatorname{EP}(f)$ or $\operatorname{GEP}(f)$ and the set of fixed points of a nonlinear map; see, for example, [10-16] and the references therein.

In 2009, Ceng et al. [17] introduced an implicit-type algorithm for finding a common element of the set of solutions of an equilibrium problem and the set of fixed points of a strict pseudo-contraction in a real Hilbert space. Recently, Kim et al. [18] and Kangtunyakarn [19] approximated a common element of the set of solutions of two generalized equilibrium problems and the set of fixed points of a strict pseudo-contraction using the shrinking projection algorithm as defined in (1.3). Quite recently, Cholamjiak and Suantai [20] established a strong convergence result regarding a system of generalized equilibrium problems and a countable family of strict pseudo-contractions in a real Hilbert space.

Inspired and motivated by the work of Nakajo and Takahashi [5], Takahashi et al. [6], Dong et al. [7], Ceng et al. [17] and Cholamjiak and Suantai [20], we propose a hybrid method based on the shrinking effect of the two half-spaces, namely $C_{n}$ and $Q_{n}$, of the underlying Hilbert space $H$. The proposed algorithm approximates a common element of the set of solutions of a finite family of generalized equilibrium problems and the set of 
common fixed points of two finite families of $k$-strict pseudo-contractions. Our results refine and improve various results announced in the current literature.

\section{Preliminaries}

Throughout the paper, we write $x_{n} \rightarrow x$ (resp. $x_{n} \rightarrow x$ ) to indicate strong convergence (resp. weak convergence). Let $H$ be a real Hilbert space, a map $P_{C}: H \rightarrow C$ defined by

$$
\left\|x-P_{C} x\right\| \leq\|x-y\| \quad \text { for all } y \in C
$$

is known as a metric projection or a nearest point projection of $H$ onto $C$. Moreover, $P_{C}$ is characterized as nonexpansive in Hilbert spaces. We know that for $x \in H$ and $z \in C$, $z=P_{C} x$ is equivalent to $\langle x-z, z-u\rangle \geq 0$ for all $u \in C$.

Let $A: C \rightarrow H$ be a $\delta$-inverse-strongly monotone map, if there exists $\delta>0$ such that

$$
\langle x-y, A x-A y\rangle \geq \delta\|A x-A y\|^{2} \quad \text { for all } x, y \in C .
$$

If $A$ is a $\delta$-inverse-strongly monotone map of $C$ onto $H$, then $A$ is $\left(\frac{1}{\delta}\right)$-Lipschitz continuous. Moreover, for $x, y \in C$ and $r>0$, we have

$$
\begin{aligned}
\|(I-r A) x-(I-r A) y\|^{2} & =\|x-y-r(A x-A y)\|^{2} \\
& =\|x-y\|^{2}-2 r\langle x-y, A x-A y\rangle+r^{2}\|A x-A y\|^{2} \\
& \leq\|x-y\|^{2}+r(r-2 \delta)\|A x-A y\|^{2} .
\end{aligned}
$$

If $r \leq 2 \delta$, then $I-r A$ is a nonexpansive map from $C$ onto $H$.

The following crucial results for a $k$-strict pseudo-contraction can be found in [21, Proposition 2.1].

Lemma 2.1 Let $C$ be a nonempty closed convex subset of a real Hilbert space $H$. If $T: C \rightarrow$ $H$ is a k-strict pseudo-contraction, then the fixed point set $F(T)$ is closed and convex so that the projection $P_{F(T)}$ is well defined.

Lemma 2.2 Let $C$ be a nonempty closed convex subset of a real Hilbert space $H$ and $T$ : $C \rightarrow C$ be a $k$-strict pseudo-contraction. Then $(I-T)$ is demiclosed, that is, if $\left\{x_{n}\right\}$ is a sequence in $C$ with $x_{n} \rightarrow x$ and $x_{n}-T x_{n} \rightarrow 0$, then $x \in F(T)$.

The following lemma is well known in the context of a real Hilbert space.

Lemma 2.3 Let $H$ be a real Hilbert space, then the following identity holds:

$$
\|\alpha x+(1-\alpha) y\|^{2}=\alpha\|x\|^{2}+(1-\alpha)\|y\|^{2}-\alpha(1-\alpha)\|x-y\|^{2} .
$$

For solving the equilibrium problem, let us assume that the bifunction $f$ satisfies the following conditions (cf. [8] and [9]):

(A1) $f(x, x)=0$ for all $x \in C$;

(A2) $f$ is monotone, i.e., $f(x, y)+f(y, x) \leq 0$ for all $x, y \in C$;

(A3) for all $x, y, z \in C, \lim \sup _{t \downarrow 0} f(t z+(1-t) x, y) \leq f(x, y)$;

(A4) $f(x, \cdot)$ is convex and lower semicontinuous for all $x \in C$. 
A central result in the theory of equilibrium problems - for an approximate solution of $\operatorname{EP}(f)$ - is the following result due to [8].

Lemma 2.4 Let $C$ be a closed convex subset of a real Hilbertspace $H$, and let $f: C \times C \rightarrow \mathbb{R}$ be a bifunction satisfying (A1)-(A4). For $r>0$ and $x \in H$, there exists $z \in C$ such that

$$
f(z, y)+\frac{1}{r}\langle y-z, z-x\rangle \geq 0 \quad \text { for all } y \in C
$$

Moreover, define a map $V_{r}: H \rightarrow C$ by

$$
V_{r}(x)=\left\{z \in C: f(z, y)+\frac{1}{r}\langle y-z, z-x\rangle \geq 0 \text { for all } y \in C\right\}
$$

for all $x \in H$. Then the following hold:

(1) $\mathrm{EP}(f)$ is closed and convex;

(2) $V_{r}$ is single-valued;

(3) $V_{r}$ is a firmly nonexpansive-type map, i.e.,

$$
\left\|V_{r} x-V_{r} y\right\|^{2} \leq\left\langle V_{r} x-V_{r} y, x-y\right\rangle \quad \text { for all } x, y \in H
$$

(4) $F\left(V_{r}\right)=\mathrm{EP}(f)$.

\section{Main result}

Let $H$ be a real Hilbert space and $C$ be its nonempty closed convex subset. Let $T_{i}(\bmod N)$, $S_{i}(\bmod N): C \rightarrow C$ be two finite families of $k$-strict pseudo-contractions such that $k=$ $\max \left\{k_{i}: 1 \leq i \leq N\right\}$. Let $f_{i}(\bmod N): C \times C \rightarrow \mathbb{R}$ be a finite family of bifunctions and $A_{i}(\bmod N): C \rightarrow H$ be a finite family of $\delta$-inverse-strongly monotone maps such that $\delta=\max \left\{\delta_{i}: 1 \leq i \leq N\right\}$.

Throughout this section, we assume that the $\bmod N$ function takes values in the indexing set $I=\{1,2,3, \ldots, N\}$ and the set of common fixed points of two finite families of $k$-strict pseudo-contractions $\left\{T_{i}\right\}_{i=1}^{N}$ and $\left\{S_{i}\right\}_{i=1}^{N}$ is nonempty, that is, $F=\left(\bigcap_{i=1}^{N} F\left(T_{i}\right)\right) \cap$ $\left(\bigcap_{i=1}^{N} F\left(S_{i}\right)\right) \neq \emptyset$.

Algorithm Our hybrid algorithm reads as follows:

$$
\begin{aligned}
& x_{1} \in C_{1}=Q_{1}=C, \\
& y_{n, i}=\beta_{n, i} z_{n, i}+\left(1-\beta_{n, i}\right) S_{i} z_{n, i}, \\
& z_{n, i}=\alpha_{n, i} u_{n, i}+\left(1-\alpha_{n, i}\right) T_{i} u_{n, i}, \\
& f_{i}\left(u_{n, i}, y\right)+\left\langle A_{i} x_{n}, y-u_{n, i}\right\rangle+\frac{1}{r_{n, i}}\left\langle y-u_{n, i}, u_{n, i}-x_{n}\right\rangle \geq 0, \quad \forall y \in C, \\
& C_{n+1}=\left\{z \in C_{n}: \sup _{i \geq 1}\left\|y_{n, i}-z\right\| \leq\left\|x_{n}-z\right\|\right\}, \\
& Q_{n+1}=\left\{z \in Q_{n}:\left\langle z-x_{n}, x_{1}-x_{n}\right\rangle \geq 0\right\}, \\
& x_{n+1}=P_{C_{n+1} \cap Q_{n+1} x_{1}, \quad n \geq 1 .}
\end{aligned}
$$


It is worth mentioning that at each iteration, algorithm (3.1) computes a projection onto intersection of two half-spaces. Since these half-spaces are closed and convex, so one can follow Dykstra's algorithm [22] for the computation of such a projection.

The main result of this section is as follows.

Theorem 3.1 Let $C$ be a nonempty closed convex subset of a real Hilbert space $H$ and let $T_{i}(\bmod N), S_{i}(\bmod N): C \rightarrow C$ be two finite families of $k$-strict pseudo-contractions. Let $f_{i}(\bmod N): C \times C \rightarrow \mathbb{R}$ be a finite family of bifunctions satisfying (A1)-(A4) and let $A_{i}(\bmod N): C \rightarrow H$ be a finite family of $\delta$-inverse-strongly monotone maps. Let $\left\{r_{n, i}\right\} \subset$ $(0, \infty)$ and $\left\{\alpha_{n, i}\right\},\left\{\beta_{n, i}\right\}$ be two control sequences such that

(C1) $0 \leq k<a \leq \alpha_{n, i}, \beta_{n, i} \leq b<1$;

(C2) $0<r<r_{n, i}<s<2 \delta$ for all $i \geq 1$.

Assume that $\mathcal{F}:=\left[\bigcap_{i=1}^{N} F\left(T_{i}\right)\right] \cap\left[\bigcap_{i=1}^{N} F\left(S_{i}\right)\right] \cap\left[\bigcap_{i=1}^{N} \operatorname{GEP}\left(f_{i}, A_{i}\right)\right] \neq \emptyset$, then the sequence $\left\{x_{n}\right\}$ generated by (3.1) converges strongly to $x=P_{\mathcal{F}} x_{1}$, where $P_{\mathcal{F}}$ is the metric projection of H onto $\mathcal{F}$.

Proof It follows from Lemma 2.4 that $u_{n, i}$ can be written as $u_{n, i}=V_{r_{n, i}}\left(x_{n}-r_{n, i} A_{i} x_{n}\right)$ for all $n \geq 1$. Moreover, it follows from the nonexpansiveness of $I-r_{n, i} A_{i}$ that

$$
\left\|u_{n, i}-p\right\| \leq\left\|x_{n}-p\right\|
$$

where $p=\bigcap_{i=1}^{N} T_{i} p=\bigcap_{i=1}^{N} S_{i} p=\bigcap_{i=1}^{N} V_{r_{n, i}}\left(p-r_{n, i} A_{i} p\right)$.

We proceed to show that algorithm (3.1) is well defined. It is obvious from the definitions of respective sets that $C_{n+1}$ is closed and $Q_{n+1}$ is closed and convex. Now, we show that $C_{n+1}$ is convex. Since $C_{1}=C$ is convex, we assume that $C_{k}$ is convex for some $k \geq 2$. For any $z \in C_{k}$, the inequality $\left\|y_{n, i}-z\right\| \leq\left\|x_{n}-z\right\|$ is equivalent to

$$
\left\|y_{n, i}\right\|^{2}-\left\|x_{n}\right\|^{2}-2\left\langle z, y_{n, i}-x_{n},\right\rangle \geq 0 \text {. }
$$

Hence, $C_{k+1}$ is convex, and consequently $C_{n+1}$ is convex for each $n \geq 1$.

Next, we show by induction that $\mathcal{F} \subset C_{n+1} \cap Q_{n+1}$ for all $n \geq 1$. Obviously, $\mathcal{F} \subset C_{1} \cap$ $Q_{1}=C$. Let $p \in \mathcal{F}$. From (2.1), (3.1) and Lemma 2.3, we have the following estimate:

$$
\begin{aligned}
\left\|z_{n, i}-p\right\|^{2}= & \left\|\alpha_{n, i}\left(u_{n, i}-p\right)+\left(1-\alpha_{n, i}\right)\left(T_{i} u_{n, i}-p\right)\right\|^{2} \\
= & \alpha_{n, i}\left\|u_{n, i}-p\right\|^{2}+\left(1-\alpha_{n, i}\right)\left\|T_{i} u_{n, i}-p\right\|^{2} \\
& -\alpha_{n, i}\left(1-\alpha_{n, i}\right)\left\|u_{n, i}-T_{i} u_{n, i}\right\|^{2} \\
\leq & \alpha_{n, i}\left\|u_{n, i}-p\right\|^{2}+\left(1-\alpha_{n, i}\right)\left(\left\|u_{n, i}-p\right\|^{2}+k\left\|u_{n, i}-T_{i} u_{n, i}\right\|^{2}\right) \\
& -\alpha_{n, i}\left(1-\alpha_{n, i}\right)\left\|u_{n, i}-T_{i} u_{n, i}\right\|^{2} \\
= & \left\|u_{n, i}-p\right\|^{2}-\left(1-\alpha_{n, i}\right)\left(\alpha_{n, i}-k\right)\left\|u_{n, i}-T_{i} u_{n, i}\right\|^{2} .
\end{aligned}
$$

Since $\alpha_{n, i}-k>0$ (by $(\mathrm{C} 1)$ ), therefore the above estimate (3.2) yields

$$
\left\|z_{n, i}-p\right\|^{2} \leq\left\|u_{n, i}-p\right\|^{2} \leq\left\|x_{n}-p\right\|^{2} .
$$


Moreover,

$$
\begin{aligned}
\left\|y_{n, i}-p\right\|^{2}= & \left\|\beta_{n, i}\left(z_{n, i}-p\right)+\left(1-\beta_{n, i}\right)\left(S_{i} z_{n, i}-p\right)\right\|^{2} \\
= & \beta_{n, i}\left\|z_{n, i}-p\right\|^{2}+\left(1-\beta_{n, i}\right)\left\|S_{i} z_{n, i}-p\right\|^{2} \\
& -\beta_{n, i}\left(1-\beta_{n, i}\right)\left\|z_{n, i}-S_{i} z_{n, i}\right\|^{2} \\
\leq & \beta_{n, i}\left\|z_{n, i}-p\right\|^{2}+\left(1-\beta_{n, i}\right)\left(\left\|z_{n, i}-p\right\|^{2}+k\left\|z_{n, i}-S_{i} z_{n, i}\right\|^{2}\right) \\
& -\beta_{n, i}\left(1-\beta_{n, i}\right)\left\|z_{n, i}-S_{i} z_{n, i}\right\|^{2} \\
\leq & \left\|z_{n, i}-p\right\|^{2}-\left(1-\beta_{n, i}\right)\left(\beta_{n, i}-k\right)\left\|z_{n, i}-S_{i} z_{n, i}\right\|^{2} .
\end{aligned}
$$

Reasoning as above and utilizing (3.3), the estimate (3.4) implies that

$$
\left\|y_{n, i}-p\right\| \leq\left\|x_{n}-p\right\|
$$

This implies that $p \in C_{n+1}$ for all $n \geq 1$. It suffices to show that $p \in Q_{n+1}$ for all $n \geq 1$. We prove this by induction. Note that $\mathcal{F} \subset Q_{1}=C$ is obvious. Assume that $\mathcal{F} \subset Q_{k}$ also $\mathcal{F} \subset$ $C_{k} \cap Q_{k}$ for some $k \geq 2$. This implies $x_{k}$ is a projection of $x_{1}$ onto $C_{k} \cap Q_{k}$, and consequently we have

$$
\left\langle x_{k}-z, x_{1}-x_{k}\right\rangle \geq 0 \quad \text { for all } z \in C_{k} \cap Q_{k} \text {. }
$$

Since $\mathcal{F} \subset C_{k} \cap Q_{k}$, we have

$$
\left\langle x_{k}-p, x_{1}-x_{k}\right\rangle \geq 0 \quad \text { for all } p \in \mathcal{F} \text {. }
$$

Hence, $p \in Q_{k+1}$, and consequently $\mathcal{F} \subset C_{n+1} \cap Q_{n+1}$ for all $n \geq 1$. Since $\mathcal{F}$ is now closed and convex, so it follows from Lemma 2.1 that $P_{\mathcal{F}}$ is well defined. Note that $x_{n+1}=P_{C_{n+1} \cap Q_{n+1}} x_{1}$, therefore $\left\|x_{n+1}-x_{1}\right\| \leq\left\|p-x_{1}\right\|$ for all $p \in \mathcal{F} \subset C_{n+1} \cap Q_{n+1}$. In particular, we have $\| x_{n+1}-$ $x_{1}\|\leq\| P_{\mathcal{F}} x_{1}-x_{1} \|$. This implies that $\left\{x_{n}\right\}$ is bounded. On the other hand, $x_{n}=P_{C_{n} \cap Q_{n}} x_{1}$ and $x_{n+1} \in C_{n+1} \cap Q_{n+1} \subset Q_{n+1}$, we have

$$
\left\|x_{n}-x_{1}\right\| \leq\left\|x_{n+1}-x_{1}\right\|
$$

That is, the sequence $\left\{\left\|x_{n}-x_{1}\right\|\right\}$ is nondecreasing. This implies $\lim _{n \rightarrow \infty}\left\|x_{n}-x_{1}\right\|$ exists. Note that

$$
\begin{aligned}
\left\|x_{n+1}-x_{n}\right\|^{2} & =\left\|x_{n+1}-x_{1}+x_{1}-x_{n}\right\|^{2} \\
& =\left\|x_{n+1}-x_{1}\right\|^{2}+\left\|x_{n}-x_{1}\right\|^{2}-2\left\langle x_{n}-x_{1}, x_{n+1}-x_{1}\right\rangle \\
& =\left\|x_{n+1}-x_{1}\right\|^{2}+\left\|x_{n}-x_{1}\right\|^{2}-2\left\langle x_{n}-x_{1}, x_{n+1}-x_{n}+x_{n}-x_{1}\right\rangle \\
& =\left\|x_{n+1}-x_{1}\right\|^{2}-\left\|x_{n}-x_{1}\right\|^{2}-2\left\langle x_{n}-x_{1}, x_{n+1}-x_{n}\right\rangle \\
& \leq\left\|x_{n+1}-x_{1}\right\|^{2}-\left\|x_{n}-x_{1}\right\|^{2} .
\end{aligned}
$$


Taking lim sup on both sides of the above estimate, we have $\lim _{\sup } \rightarrow \infty\left\|x_{n+1}-x_{n}\right\|^{2}=0$. That is,

$$
\lim _{n \rightarrow \infty}\left\|x_{n+1}-x_{n}\right\|=0 .
$$

Since $x_{n+1} \in C_{n+1}$, we have $\left\|y_{n, i}-x_{n+1}\right\| \leq\left\|x_{n}-x_{n+1}\right\|$. This implies

$$
\lim _{n \rightarrow \infty}\left\|y_{n, i}-x_{n+1}\right\|=0 .
$$

Moreover, it follows from (3.6), (3.7) and the following inequality:

$$
\left\|y_{n, i}-x_{n}\right\| \leq\left\|y_{n, i}-x_{n+1}\right\|+\left\|x_{n+1}-x_{n}\right\|
$$

that

$$
\lim _{n \rightarrow \infty}\left\|y_{n, i}-x_{n}\right\|=0
$$

Note that

$$
\begin{aligned}
\left\|u_{n, i}-p\right\|^{2} & =\left\|V_{r_{n, i}}\left(I-r_{n, i} A_{i}\right) x_{n}-V_{r_{n, i}}\left(I-r_{n, i} A_{i}\right) p\right\|^{2} \\
& =\left\|\left(I-r_{n, i} A_{i}\right) x_{n}-\left(I-r_{n, i} A_{i}\right) p\right\|^{2} \\
& =\left\|x_{n}-p-r_{n, i}\left(A_{i} x_{n}-A_{i} p\right)\right\|^{2} \\
& =\left\|x_{n}-p\right\|^{2}-2 r_{n, i}\left(A_{i} x_{n}-A_{i} p, x_{n}-p\right\rangle+r_{n, i}^{2}\left\|A_{i} x_{n}-A_{i} p\right\|^{2} \\
& \leq\left\|x_{n}-p\right\|^{2}+r_{n, i}\left(r_{n, i}-2 \delta\right)\left\|A_{i} x_{n}-A_{i} p\right\|^{2} .
\end{aligned}
$$

Since $\left\|y_{n, i}-p\right\|^{2} \leq\left\|z_{n, i}-p\right\|^{2} \leq\left\|u_{n, i}-p\right\|^{2}$, therefore utilizing (3.9), we get

$$
\left\|y_{n, i}-p\right\|^{2} \leq\left\|x_{n}-p\right\|^{2}-r_{n, i}\left(2 \delta-r_{n, i}\right)\left\|A_{i} x_{n}-A_{i} p\right\|^{2} .
$$

Re-arranging the terms in the above estimate and utilizing (C2), we have

$$
r(2 \delta-s)\left\|A_{i} x_{n}-A_{i} p\right\|^{2} \leq\left\|x_{n}-p\right\|^{2}-\left\|y_{n, i}-p\right\|^{2} \leq\left(\left\|x_{n}-p\right\|+\left\|y_{n, i}-p\right\|\right)\left\|x_{n}-y_{n, i}\right\| .
$$

Hence, (3.8) implies that

$$
\lim _{n \rightarrow \infty}\left\|A_{i} x_{n}-A_{i} p\right\|=0 \quad \text { for all } i \geq 1 .
$$

Since $k<a \leq \beta_{n, i} \leq b<1$, then the following variant of the estimate (3.4) implies that

$$
\begin{aligned}
(1-b)(a-k)\left\|z_{n, i}-S_{i} z_{n, i}\right\|^{2} & \leq\left\|x_{n}-p\right\|^{2}-\left\|y_{n, i}-p\right\|^{2} \\
& \leq\left(\left\|x_{n}-p\right\|+\left\|y_{n, i}-p\right\|\right)\left\|x_{n}-y_{n, i}\right\| .
\end{aligned}
$$

Again, utilizing (3.8), we have

$$
\lim _{n \rightarrow \infty}\left\|z_{n, i}-S_{i} z_{n, i}\right\|=0 \quad \text { for all } i \geq 1 .
$$


Observe that $\left\|y_{n, i}-z_{n, i}\right\|=\left(1-\beta_{n, i}\right)\left\|S_{i} z_{n, i}-z_{n, i}\right\|$. It follows from the fact that $k<a \leq \beta_{n, i} \leq$ $b<1$ and (3.11) that

$$
\lim _{n \rightarrow \infty}\left\|y_{n, i}-z_{n, i}\right\|=0 \quad \text { for all } i \geq 1 .
$$

Moreover, $\left\|x_{n}-z_{n, i}\right\| \leq\left\|x_{n}-y_{n, i}\right\|+\left\|y_{n, i}-z_{n, i}\right\|$. Letting $n \rightarrow \infty$ on both sides and utilizing (3.8) and (3.12), we have

$$
\lim _{n \rightarrow \infty}\left\|x_{n}-z_{n, i}\right\|=0 \quad \text { for all } i \geq 1 .
$$

Reasoning as above, that is, $k<a \leq \alpha_{n, i} \leq b<1$, we consider the following variant of the estimate (3.2):

$$
\begin{aligned}
(1-b)(a-k)\left\|u_{n, i}-T_{i} u_{n, i}\right\|^{2} & \leq\left\|x_{n}-p\right\|^{2}-\left\|z_{n, i}-p\right\|^{2} \\
& \leq\left(\left\|x_{n}-p\right\|+\left\|z_{n, i}-p\right\|\right)\left\|x_{n}-z_{n, i}\right\| .
\end{aligned}
$$

Hence, we conclude from the above estimate and (3.13) that

$$
\lim _{n \rightarrow \infty}\left\|u_{n, i}-T_{i} u_{n, i}\right\|=0 \quad \text { for all } i \geq 1
$$

On the other hand, $\left\|z_{n, i}-u_{n, i}\right\|=\left(1-\alpha_{n, i}\right)\left\|T_{i} u_{n, i}-u_{n, i}\right\|$. Making use of the fact that $k<$ $a \leq \alpha_{n, i} \leq b<1$ and (3.14), we get

$$
\lim _{n \rightarrow \infty}\left\|z_{n, i}-u_{n, i}\right\|=0 \quad \text { for all } i \geq 1 .
$$

Moreover, we conclude from the estimates (3.12) and (3.15) that

$$
\lim _{n \rightarrow \infty}\left\|y_{n, i}-u_{n, i}\right\|=0 \quad \text { for all } i \geq 1 .
$$

As a direct consequence of the estimates (3.8) and (3.16), we have

$$
\lim _{n \rightarrow \infty}\left\|x_{n}-u_{n, i}\right\|=0 \quad \text { for all } i \geq 1
$$

Next, we show that $\omega\left(x_{n}\right) \subset \mathcal{F}$, where $\omega\left(x_{n}\right)$ is the set of all weak $\omega$-limit of $\left\{x_{n}\right\}$. Since $\left\{x_{n}\right\}$ is bounded, therefore $\omega\left(x_{n}\right) \neq \emptyset$. Let $q \in \omega\left(x_{n}\right)$, there exists a subsequence $\left\{x_{n_{j}}\right\}$ of $\left\{x_{n}\right\}$ such that $x_{n_{j}} \rightarrow q$. It follows from the estimate (3.17) that $u_{n_{j}, i} \rightarrow q$. We first show that $q \in \operatorname{GEP}\left(f_{1}, A_{1}\right)$, where $f_{1}=f_{n_{j}}$ for some $j \geq 1$. From $u_{n_{j}, i}=V_{r_{n_{j}, i}}\left(I-r_{n_{j}, i} A_{i}\right) x_{n}$, for all $n \geq 1$, we have

$$
f_{1}\left(u_{n_{j}, i}, y\right)+\left\langle A_{1} x_{n_{j}}, y-u_{n_{j}, i}\right\rangle+\frac{1}{r_{n_{j}, i}}\left\langle y-u_{n_{j}, i}, u_{n_{j}, i}-x_{n_{j}}\right\rangle \geq 0 \quad \text { for all } y \in C .
$$

From (A2), we have

$$
\left\langle A_{1} x_{n_{j}}, y-u_{n_{j}, i}\right\rangle+\frac{1}{r_{n_{j}, i}}\left\langle y-u_{n_{j}, i}, u_{n_{j}, i}-x_{n_{j}}\right\rangle \geq f_{1}\left(y, u_{n_{j}, i}\right) \quad \text { for all } y \in C
$$


Let $y_{t}=t y+(1-t) q$ for $0<t<1$ and $y \in C$. Since $q \in C$, this implies that $y_{t} \in C$. It follows from the estimate (3.18) that

$$
\begin{aligned}
\left\langle y_{t}-u_{n_{j}, i}, A_{1} y_{t}\right\rangle \geq & \left\langle y_{t}-u_{n_{j}, i}, A_{1} y_{t}\right\rangle-\left\langle A_{1} x_{n_{j}}, y_{t}-u_{n_{j}, i}\right\rangle \\
& -\left\langle y_{t}-u_{n_{j}, i}, \frac{u_{n_{j}, i}-x_{n_{j}}}{r_{n_{j}, i}}\right\rangle+f_{1}\left(y_{t}, u_{n_{j}, i}\right) \\
= & \left\langle y_{t}-u_{n_{j}, i}, A_{1} y_{t}-A_{1} u_{n_{j}, i}\right\rangle+\left\langle y_{t}-u_{n_{j}, i}, A_{1} u_{n_{j}, i}-A_{1} x_{n_{j}}\right\rangle \\
& -\left\langle y_{t}-u_{n_{j}, i}, \frac{u_{n_{j}, i}-x_{n_{j}}}{r_{n_{j}, i}}\right\rangle+f_{1}\left(y_{t}, u_{n_{j}, i}\right) .
\end{aligned}
$$

Since $\lim _{n \rightarrow \infty}\left\|x_{n_{j}}-u_{n_{j}, i}\right\|=0$, therefore $\lim _{n \rightarrow \infty}\left\|A_{1} x_{n_{j}}-A_{1} u_{n_{j}, i}\right\|=0$. Moreover, it follows from the monotonicity of $A$ that $\left\langle y_{t}-u_{n_{j}, i}, A_{1} y_{t}-A_{1} u_{n_{j}, i}\right\rangle \geq 0$. Hence, (A4) implies that

$$
\left\langle y_{t}-q, A_{1} y_{t}\right\rangle \geq f_{1}\left(y_{t}, q\right) .
$$

Using (3.20), (A1) and (A4), the following estimate:

$$
\begin{aligned}
0 & =f_{1}\left(y_{t}, y_{t}\right) \leq t f_{1}\left(y_{t}, y\right)+(1-t) f_{1}\left(y_{t}, q\right) \\
& \leq t f_{1}\left(y_{t}, y\right)+(1-t)\left\langle y_{t}-q, A_{1} y_{t}\right\rangle \\
& =t f_{1}\left(y_{t}, y\right)+(1-t) t\left\langle y-q, A_{1} y_{t}\right\rangle,
\end{aligned}
$$

implies that

$$
f_{1}\left(y_{t}, y\right)+(1-t)\left\langle y-q, A_{1} y_{t}\right\rangle \geq 0 \text {. }
$$

Letting $t \rightarrow 0$, we have $f_{1}(q, y)+\left\langle y-q, A_{1} q\right\rangle \geq 0$ for all $y \in C$. Thus, $q \in \operatorname{GEP}\left(f_{1}, A_{1}\right)$. In a similar fashion, we have some $k \geq 1$ such that $f_{2}=f_{n_{k}}$ and $q \in \operatorname{GEP}\left(f_{2}, A_{2}\right)$. Therefore, $q \in \bigcap_{i=1}^{N} \operatorname{GEP}\left(f_{i}, A_{i}\right)$. Since $u_{n_{j}, i} \rightarrow q$, so it follows from (3.14) and Lemma 2.2 that $q \in \bigcap_{i=1}^{N} F\left(T_{i}\right)$. Reasoning as above, we can show that $q \in \bigcap_{i=1}^{N} F\left(S_{i}\right)$. Hence, $q \in \mathcal{F}$. Let $x=P_{\mathcal{F} x_{1}}$, which implies that $x=P_{\mathcal{F}} x_{1} \in C_{n+1}$. Since $x_{n+1}=P_{C_{n+1} \cap Q_{n+1}} x_{1} \in C_{n+1}$, we have

$$
\left\|x_{n+1}-x_{1}\right\| \leq\left\|x-x_{1}\right\| .
$$

On the other hand, we have

$$
\begin{aligned}
\left\|x-x_{1}\right\| & \leq\left\|q-x_{1}\right\| \\
& \leq \liminf _{j \rightarrow \infty}\left\|x_{n_{j}}-x_{1}\right\| \\
& \leq \limsup _{j \rightarrow \infty}\left\|x_{n_{j}}-x_{1}\right\| \\
& \leq\left\|x-x_{1}\right\| .
\end{aligned}
$$

That is,

$$
\left\|q-x_{1}\right\|=\lim _{j \rightarrow \infty}\left\|x_{n_{j}}-x_{1}\right\|=\left\|x-x_{1}\right\| .
$$


Therefore, we conclude that $\lim _{j \rightarrow \infty} x_{n_{j}}=q=P_{\mathcal{F}} x_{1}$. From the arbitrariness of $\left\{x_{n_{j}}\right\}$, we get that $\lim _{n \rightarrow \infty} x_{n}=P_{\mathcal{F}} x_{1}$. This completes the proof.

In particular, if $T_{i}$ and $S_{i}$ - in algorithm (3.1) - are two finite families of nonexpansive maps, then the following result holds.

Corollary 3.2 Let $C$ be a nonempty closed convex subset of a real Hilbert space $H$ and let $T_{i}(\bmod N), S_{i}(\bmod N): C \rightarrow C$ be two finite families of nonexpansive maps. Let $f_{i}(\bmod N):$ $C \times C \rightarrow \mathbb{R}$ be a finite family of bifunctions satisfying (A1)-(A4) and let $A_{i}(\bmod N): C \rightarrow H$ be a finite family of $\delta$-inverse-strongly monotone maps. Let $\left\{r_{n, i}\right\} \subset(0, \infty)$ and $\left\{\alpha_{n, i}\right\},\left\{\beta_{n, i}\right\}$ be two control sequences such that

(C1) $0 \leq k<a \leq \alpha_{n, i}, \beta_{n, i} \leq b<1$;

(C2) $0<r<r_{n, i}<s<2 \delta$ for all $i \geq 1$.

Assume that $\mathcal{F}:=\left[\bigcap_{i=1}^{N} F\left(T_{i}\right)\right] \cap\left[\bigcap_{i=1}^{N} F\left(S_{i}\right)\right] \cap\left[\bigcap_{i=1}^{N} \operatorname{GEP}\left(f_{i}, A_{i}\right)\right] \neq \emptyset$, then the sequence $\left\{x_{n}\right\}$ generated by (3.1) converges strongly to $x=P_{\mathcal{F}} x_{1}$, where $P_{\mathcal{F}}$ is the metric projection of H onto $\mathcal{F}$.

In order to address variational inequality problems coupled with the fixed point problems, we prove the following result with a slight modification of algorithm (3.1).

Theorem 3.3 Let $C$ be a nonempty closed convex subset of a real Hilbert space $H$ and let $T_{i}(\bmod N), S_{i}(\bmod N): C \rightarrow C$ be two finite families of $k$-strict pseudo-contractions. Let $f_{i}(\bmod N): C \times C \rightarrow \mathbb{R}$ be a finite family of bifunctions satisfying (A1)-(A4) and let $A_{i}(\bmod N): C \rightarrow H$ be a finite family of $\delta$-inverse-strongly monotone maps. Let $\left\{r_{n, i}\right\} \subset$ $(0, \infty)$ and $\left\{\alpha_{n, i}\right\},\left\{\beta_{n, i}\right\}$ be two control sequences such that

(C1) $0 \leq k<a \leq \alpha_{n, i}, \beta_{n, i} \leq b<1$;

(C2) $0<r<r_{n, i}<s<2 \delta$ for all $i \geq 1$.

Assume that $\mathcal{F}:=\left[\bigcap_{i=1}^{N} F\left(T_{i}\right)\right] \cap\left[\bigcap_{i=1}^{N} F\left(S_{i}\right)\right] \cap\left[\bigcap_{i=1}^{N} \mathrm{VI}\left(C, A_{i}\right)\right] \neq \emptyset$, then the sequence $\left\{x_{n}\right\}$ generated by

$$
\begin{aligned}
& x_{1} \in C_{1}=Q_{1}=C, \\
& h_{n, i}=P_{C}\left(I-r_{n, i} A_{i}\right) x_{n}, \\
& z_{n, i}=\alpha_{n, i} h_{n, i}+\left(1-\alpha_{n, i}\right) T_{i} h_{n, i}, \\
& y_{n, i}=\beta_{n, i} z_{n, i}+\left(1-\beta_{n, i}\right) S_{i} z_{n, i}, \\
& C_{n+1}=\left\{z \in C_{n}: \sup _{i \geq 1}\left\|y_{n, i}-z\right\| \leq\left\|x_{n}-z\right\|\right\}, \\
& Q_{n+1}=\left\{z \in Q_{n}:\left\langle z-x_{n}, x_{1}-x_{n}\right\rangle \geq 0\right\}, \\
& x_{n+1}=P_{C_{n+1} \cap Q_{n+1} x_{1}, \quad n \geq 1,}
\end{aligned}
$$

converges strongly to $x=P_{\mathcal{F}} x_{1}$, where $P_{\mathcal{F}}$ is the metric projection of $H$ onto $\mathcal{F}$.

Proof Set $f_{i}(x, y) \equiv 0$ for each $i \geq 1$, then

$$
\left\langle A_{i} x_{n}, y-u_{n, i}\right\rangle+\frac{1}{r_{n, i}}\left\langle y-u_{n, i}, u_{n, i}-x_{n}\right\rangle \geq 0
$$


is equivalent to

$$
\left\langle x_{n}-r_{n, i} A_{i} x_{n}-u_{n, i}, u_{n, i}-y\right\rangle \geq 0 .
$$

This implies that $h_{n, i}=u_{n, i}:=P_{C}\left(x_{n}-r_{n, i} A_{i} x_{n}\right)$. The desired result then follows from Theorem 3.1 immediately.

As an application of Theorem 3.1 - by substituting $A_{i} \equiv 0$ for all $i \geq 1$ in algorithm (3.1) - we have the following result for a finite family of equilibrium problems.

Theorem 3.4 Let $C$ be a nonempty closed convex subset of a real Hilbert space $H$ and let $T_{i}(\bmod N), S_{i}(\bmod N): C \rightarrow C$ be two finite families of $k$-strict pseudo-contractions. Let $f_{i}(\bmod N): C \times C \rightarrow \mathbb{R}$ be a finite family of bifunctions satisfying (A1)-(A4). Let $\left\{\alpha_{n, i}\right\},\left\{\beta_{n, i}\right\}$ be two control sequences such that

(C1) $0 \leq k<a \leq \alpha_{n, i}, \beta_{n, i} \leq b<1$.

Assume that $\mathcal{F}:=\left[\bigcap_{i=1}^{N} F\left(T_{i}\right)\right] \cap\left[\bigcap_{i=1}^{N} F\left(S_{i}\right)\right] \cap\left[\bigcap_{i=1}^{N} \operatorname{EP}\left(f_{i}\right)\right] \neq \emptyset$, then the sequence $\left\{x_{n}\right\}$ generated by

$$
\begin{aligned}
& x_{1} \in C_{1}=Q_{1}=C, \\
& f_{i}\left(u_{n, i}, y\right)+\frac{1}{r_{n, i}}\left\langle y-u_{n, i}, u_{n, i}-x_{n}\right\rangle \geq 0, \quad \forall y \in C, \\
& y_{n, i}=\beta_{n, i} z_{n, i}+\left(1-\beta_{n, i}\right) S_{i} z_{n, i}, \\
& z_{n, i}=\alpha_{n, i} u_{n, i}+\left(1-\alpha_{n, i}\right) T_{i} u_{n, i}, \\
& C_{n+1}=\left\{z \in C_{n}: \sup _{i \geq 1}\left\|y_{n, i}-z\right\| \leq\left\|x_{n}-z\right\|\right\}, \\
& Q_{n+1}=\left\{z \in Q_{n}:\left\langle z-x_{n}, x_{1}-x_{n}\right\rangle \geq 0\right\}, \\
& x_{n+1}=P_{C_{n+1} \cap Q_{n+1}} x_{1}, \quad n \geq 1,
\end{aligned}
$$

converges strongly to $x=P_{\mathcal{F}} x_{1}$, where $P_{\mathcal{F}}$ is the metric projection of $H$ onto $\mathcal{F}$.

Remark 3.5 Additionally - in Theorem 3.4 - if we set $\beta_{n, i} \equiv 0$, then Theorem 3.4 sets analogue [17, Theorem 3.3] in the following aspects:

(i) from a single $k$-strict pseudo-contraction to a finite family of maps;

(ii) from an equilibrium problem to a finite family of generalized equilibrium problems.

Competing interests

The authors declare that they have no competing interests.

Authors' contributions

All authors contributed equally and significantly in writing this paper. All authors read and approved the final manuscript.

\section{Author details}

'Department of Mathematics, Technische Universitat Darmstadt, Schlossgartenstrasse 7, Darmstadt, 64289, Germany. ${ }^{2}$ Department of Mathematics, The Islamia University of Bahawalpur, Bahawalpur, 63100, Pakistan. ${ }^{3}$ Department of Mathematics and Statistics, King Fahd University of Petroleum and Minerals, Dhahran, 3126, Saudi Arabia. 


\section{Acknowledgements}

We wish to thanks the referees for careful reading and helpful comments which led the manuscript to the present form. The author M.A.A. Khan gratefully acknowledges the support from German Science Foundation (DFG Project KO 1737/5-1) and Higher Education Commission of Pakistan. The author H. Fukhar-ud-din is grateful to King Fahd University of Petroleum \& Minerals for support during this research.

Received: 28 June 2012 Accepted: 22 January 2013 Published: 11 February 2013

\section{References}

1. Browder, FE, Petryshyn, WV: Construction of fixed points of nonlinear mappings in Hilbert space. J. Math. Anal. Appl. 20, 197-228 (1967)

2. Chidume, CE, Mutangadura, SA: An example on the Mann iteration method for Lipschitz pseudocontractions. Proc. Am. Math. Soc. 129, 2359-2363 (2001)

3. Haugazeau, Y: Sur les inéquations variationnelles et la minimisation de fonctionnelles convexes. Thèse, Université de Paris, Paris, France (1968)

4. Solodov, MV, Svaiter, BF: Forcing strong convergence of proximal point iterations in a Hilbert space. Math. Program. Ser. A 87, 189-202 (2000)

5. Nakajo, K, Takahashi, W: Strong convergence theorems for nonexpansive mappings and nonexpansive semigroups. J. Math. Anal. Appl. 279, 372-379 (2003)

6. Takahashi, W, Takeuchi, Y, Kubota, R: Strong convergence theorems by hybrid methods for families of nonexpansive mappings in Hilbert spaces. J. Math. Anal. Appl. 341, 276-286 (2008)

7. Dong, Q-L, He, S, Su, F: Strong convergence theorems by shrinking projection methods for class $\tau$-mappings. Fixed Point Theory Appl. 2011, Article ID 681214 (2011)

8. Blum, E, Oettli, W: From optimization and variational inequalities to equilibrium problems. Math. Stud. 63, 123-145 (1994)

9. Combettes, PL, Hirstoaga, SA: Equilibrium programming in Hilbert spaces. J. Nonlinear Convex Anal. 6, 117-136 (2005)

10. Moudafi, A: Second order differential proximal methods for equilibrium problems. J. Inequal. Pure Appl. Math. 4 Article ID 18 (2003)

11. Cholamjiak, P, Suantai, S: A new hybrid algorithm for variational inclusions, generalized equilibrium problems and a finite family of quasi-nonexpansive mappings. Fixed Point Theory Appl. 2009, Article ID 350979 (2009) doi:10.1155/2009/350979

12. Cholamjiak, P, Suantai, S: Convergence analysis for a system of equilibrium problems and a countable family of relatively quasi-nonexpansive mappings in Banach spaces. Abstr. Appl. Anal. 2010, Article ID 141376 (2010) doi:10.1155/2010/141376

13. Cholamjiak, P, Suantai, S: An iterative method for equilibrium problems and a finite family of relatively nonexpansive mappings in a Banach Space. Appl. Math. Comput. 217, 3825-3831 (2010)

14. Cholamjiak, P, Suantai, S: A hybrid method for a family of relatively quasi-nonexpansive mappings and an equilibrium problem in Banach spaces. J. Glob. Optim. (2011). doi:10.1007/s10898-011-9743-9

15. Tada, A, Takahashi, W: Weak and strong convergence theorems for a nonexpansive mapping and an equilibrium problem. J. Optim. Theory Appl. 133, 359-370 (2007)

16. Takahashi, S, Takahashi, W: Strong convergence theorem for a generalized equilibrium problem and a nonexpansive mapping in a Hilbert space. Nonlinear Anal. 69, 1025-1033 (2008)

17. Ceng, L-C, Al-Homidan, S, Ansari, QH, Yao, J-C: An iterative scheme for equilibrium problems and fixed point problems of strict pseudo-contraction mappings. J. Comput. Appl. Math. 223, 967-974 (2009)

18. Kim, JK, Cho, SY, Qin, X: Hybrid projection algorithms for generalized equilibrium problems and strictly pseudo-contractive mappings. J. Inequal. Appl. 2011, Article ID 312602 (2010)

19. Kangtunyakarn, A: Hybrid algorithm for finding common elements of the set of generalized equilibrium problems and the set of fixed point problems of strictly pseudo-contractive mapping. Fixed Point Theory Appl. 2011, Article ID 274820 (2011)

20. Cholamjiak, P, Suantai, S: Convergence analysis for a system of generalized equilibrium problems and a countable family of strict pseudo contractions. Fixed Point Theory Appl. 2011, Article ID 941090 (2011)

21. Marino, G, Xu, H-K: Weak and strong convergence theorems for strict pseudo-contractions in Hilbert spaces. J. Math. Anal. Appl. 329, 336-346 (2007)

22. Boyle, JP, Dykstra, RL: A method for finding projections onto the intersection of convex sets in Hilbert spaces. In: Advances in Order Restricted Statistical Inference. Lecture Notes in Statistics, vol. 37, pp. 28-47. Springer, Berlin (1986)

doi:10.1186/1687-1812-2013-30

Cite this article as: Khan and Fukhar-ud-din: Strong convergence by the shrinking effect of two half-spaces and applications. Fixed Point Theory and Applications 2013 2013:30. 